\title{
Does the GPM mission improve the systematic error component in satellite rainfall estimates over TRMM? An evaluation at a pan-India scale
}

\author{
Harsh Beria ${ }^{1}$, Trushnamayee Nanda ${ }^{2}$, Deepak Singh Bisht ${ }^{2}$, and Chandranath Chatterjee ${ }^{2}$ \\ ${ }^{1}$ Institute of Earth Surface Dynamics, University of Lausanne, Lausanne, Switzerland \\ ${ }^{2}$ Agricultural and Food Engineering Department, Indian Institute of Technology Kharagpur, Kharagpur, India
}

Correspondence to: Harsh Beria (harsh.beria@unil.ch)

Received: 11 May 2016 - Discussion started: 27 June 2016

Revised: 28 September 2017 - Accepted: 5 October 2017 - Published: 1 December 2017

\begin{abstract}
The last couple of decades have seen the outburst of a number of satellite-based precipitation products with Tropical Rainfall Measuring Mission (TRMM) as the most widely used for hydrologic applications. Transition of TRMM into the Global Precipitation Measurement (GPM) promises enhanced spatio-temporal resolution along with upgrades to sensors and rainfall estimation techniques. The dependence of systematic error components in rainfall estimates of the Integrated Multi-satellitE Retrievals for GPM (IMERG), and their variation with climatology and topography, was evaluated over 86 basins in India for year 2014 and compared with the corresponding (2014) and retrospective (1998-2013) TRMM estimates. IMERG outperformed TRMM for all rainfall intensities across a majority of Indian basins, with significant improvement in low rainfall estimates showing smaller negative biases in 75 out of 86 basins. Low rainfall estimates in TRMM showed a systematic dependence on basin climatology, with significant overprediction in semiarid basins, which gradually improved in the higher rainfall basins. Medium and high rainfall estimates of TRMM exhibited a strong dependence on basin topography, with declining skill in higher elevation basins. The systematic dependence of error components on basin climatology and topography was reduced in IMERG, especially in terms of topography. Rainfall-runoff modeling using the Variable Infiltration Capacity (VIC) model over two flood-prone basins (Mahanadi and Wainganga) revealed that improvement in rainfall estimates in IMERG did not translate into improvement in runoff simulations. More studies are required over basins in different hydroclimatic zones to evaluate the hydrologic significance of IMERG.
\end{abstract}

\section{Introduction}

The developing part of the world suffers from an acute shortage of hydrologic data, both in terms of quality and quantity. A recent commentary from Mujumdar (2015) provided insights into the problems faced by the Indian hydrologic community due to the lack of willingness of the relevant governmental bodies to openly share meteorologic and hydrologic data and its metadata to the research community. With the threats of climate change looming large, high-quality precipitation products (in terms of accuracy and spatio-temporal resolution) are the need of the hour to analyze hydro-meteorological processes in real time. Satellite precipitation products offer a viable alternative to gaugebased rainfall estimates.

A number of satellite-based precipitation estimates have cropped up in the past two decades, the famous ones being Climate Prediction Center morphing technique (CMORPH), Precipitation Estimation from Remotely Sensed Information Using Artificial Neural Networks (PERSIANN), PERSIANN Climate Data Record (PERSIANN-CDR), Tropical Rainfall Measuring Mission (TRMM), Asian Precipitation - Highly-Resolved Observational Data Integration Towards Evaluation (APHRODITE) and National Oceanic and Atmospheric Administration (NOAA) Climate Prediction Center (CPC). A number of studies over the past decade have evaluated the hydrologic application of these datasets over regions with varied topography and climatology.

Artan et al. (2007) found reasonable streamflow simulations using CPC over four basins in Africa and Southeast Asia while Collischonn et al. (2008) found similar re- 
sults using TRMM over the Amazon River basin. Akhtar et al. (2009) used neural networks to forecast discharges at varying lead times using TRMM 3B42V6 precipitation estimates. Wu et al. (2012) used TRMM 3B42V6 estimates to develop a real-time flood monitoring system and concluded that the probability of detection (POD) improved with longer flood durations and larger affected areas. Kneis et al. (2014) evaluated TRMM 3B42V7 and its real-time counterpart TRMM 3B42V7RT over the Mahanadi River basin in India and found the research product (3B42) to be superior to the real-time alternative (3B42RT). Peng et al. (2014) found a systematic dependence of TRMM estimates on climatology in northwest China, characterizing the wetter regions better than the drier ones. Bajracharya et al. (2014) used CPC to drive a hydrologic model over the Bagmati basin in Nepal and reported that the incorporation of local rain gauge data tremendously benefited the streamflow simulations. Shah and Mishra (2016) explored uncertainty in the estimates of multiple satellite rainfall products over major Indian basins. Most of the studies which evaluated multiple satellite precipitation estimates have reported TRMM to give the best estimate over the tropical part of the world (Gao and Liu, 2013; Prakash et al., 2016b; Zhu et al., 2016).

The TRMM satellite was launched in late 1997 and provides high-resolution $\left(0.25^{\circ} \times 0.25^{\circ}\right)$ quasi-global $\left(50^{\circ} \mathrm{N}-\mathrm{S}\right)$ rainfall estimates (Huffman et al., 2007). The TRMM mission is a joint mission between the National Aeronautics and Space Administration (NASA) and the Japan Aerospace Exploration Agency (JAXA) to study rainfall for weather and climate research. The TRMM satellite produced 17 years of valuable precipitation data over the tropics.

Owing to the tremendous success of the TRMM Multisatellite Precipitation Analysis (TMPA) mission, Global Precipitation Measurement (GPM) was launched on 27 February 2014 (Liu, 2016). The GPM sensors carry the first spaceborne dual-frequency phased array precipitation radar (DPR) operating at $\mathrm{Ku}(13 \mathrm{GHz})$ and $\mathrm{Ka}(35 \mathrm{GHz})$ bands and a canonical-scanning multichannel $(10-183 \mathrm{GHz})$ microwave imager (GMI) (Hou et al., 2014). The improved sensitivity of the $\mathrm{Ku}$ and $\mathrm{Ka}$ bands allows for improved detection of low precipitation rates $\left(<0.5 \mathrm{~mm} \mathrm{~h}^{-1}\right)$ and falling snow.

A few preliminary assessments of GPM over India and China (Prakash et al., 2016a, b; Tang et al., 2016a) suggest an improvement over TMPA. For the 2014 monsoon, Prakash et al., (2016b) reported that Integrated Multi-satellitE Retrievals for GPM (IMERG), which is a level three multisatellite precipitation algorithm of GPM (Hou et al., 2014), outperformed TMPA in extreme rainfall detection along the Himalayan foothills in north India and over northwestern India, with slightly reduced false alarms. Tang et al. (2016a) found that IMERG outperformed TMPA in almost all the indices for every sub-region of mainland China at 3-hourly and daily temporal resolutions. They also reported that IMERG reproduced probability density functions more accurately at various precipitation intensities and better represented the precipitation diurnal cycles. In another work by Prakash et al. (2016a), IMERG was compared with Global Satellite Mapping of Precipitation (GSMaP) V6 and TMPA 3B42V7 for the 2014 monsoon over India. It was found that IMERG estimates represented the mean monsoon rainfall and its variability more realistically, with fewer missed and false precipitation bias and improvements in the precipitation distribution over low rainfall rates.

Most of the previous studies that compared pan-India satellite and reanalysis precipitation products focused on a grid scale rather than a basin scale (Prakash et al., 2015, 2016a, b). We followed a basin-scale approach as it is more relevant in terms of water resources assessment for policy makers. It provides a clear signal of the utility of the satellite precipitation products at the required spatial resolution for water managers working at a basin scale. Also, at a basin scale, the statistical and hydrologic results are more complementary (Bisht et al., 2017; Kneis et al., 2014).

In this study, we comprehensively evaluated TRMM 3B42 from 1998 to 2013 over 86 basins in India and explored systematic biases due to climatology and topography. We then compared TRMM 3B42 precipitation estimates with IMERG for 2014 and explored if the systematic biases were reduced in IMERG, and whether IMERG was able to better capture the low rainfall magnitudes. Finally, we used a macroscale hydrologic model (Variable Infiltration Capacity, VIC) to evaluate TRMM and IMERG over two flood prone basins in eastern India (Hirakud catchment of the Mahanadi River basin and the Wainganga catchment of the Godavari River basin) for the year 2014 .

\section{Description of the study area, datasets used and methodology}

\subsection{Study area}

The Water Resources Information System of India (IndiaWRIS) delineates India into multiple sub-basins (Fig. 1a; Government of India, 2014). In this study, 86 basins were used, with the five excluded basins located in the Jammu and Kashmir region of northern India (details included in Table S1 in the Supplement). Also, the Lakshadweep islands (located off the Indian west coast in the Arabian Sea) and the Andaman and Nicobar islands (located in the Bay of Bengal) were excluded from the analysis due to scarce rain-gauge monitoring network data.

Most of India experiences a tropical monsoon type of climate receiving an average annual rainfall of around $1100 \mathrm{~mm} \mathrm{year}^{-1}$, of which about $70-80 \%$ is concentrated during the monsoon season (June-September). Figure 2a shows the spatial distribution of rainfall (details in Table S1), calculated using the India Meteorological Department (IMD) gridded precipitation dataset (computed using 31 years, 1980-2010, of rainfall time series) over India. The 

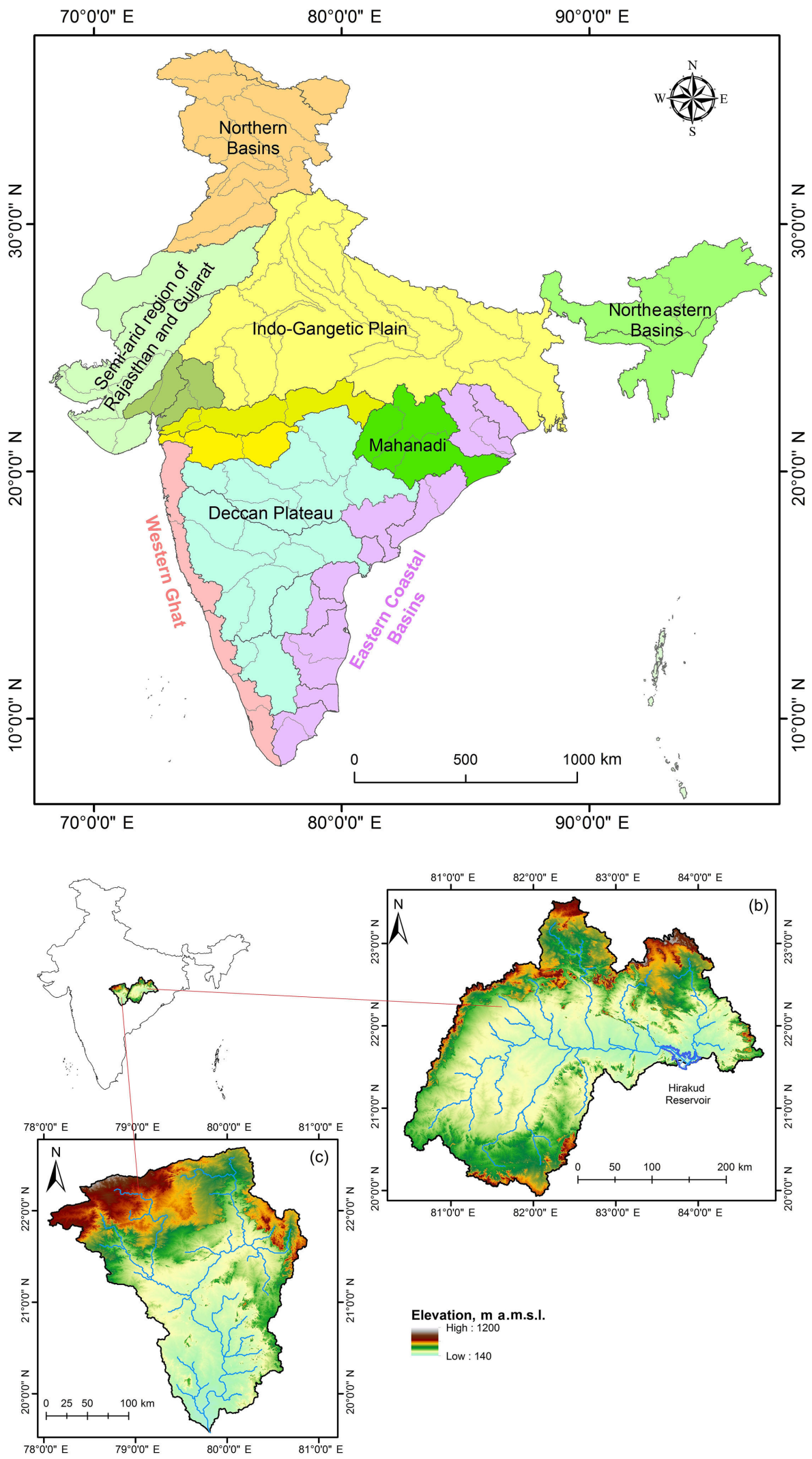

Figure 1. (a) Map of the major basins in India including west and east flowing rivers, (b) map of the Hirakud catchment of the Mahanadi River basin and (c) Wainganga catchment of the Godavari River basin. 


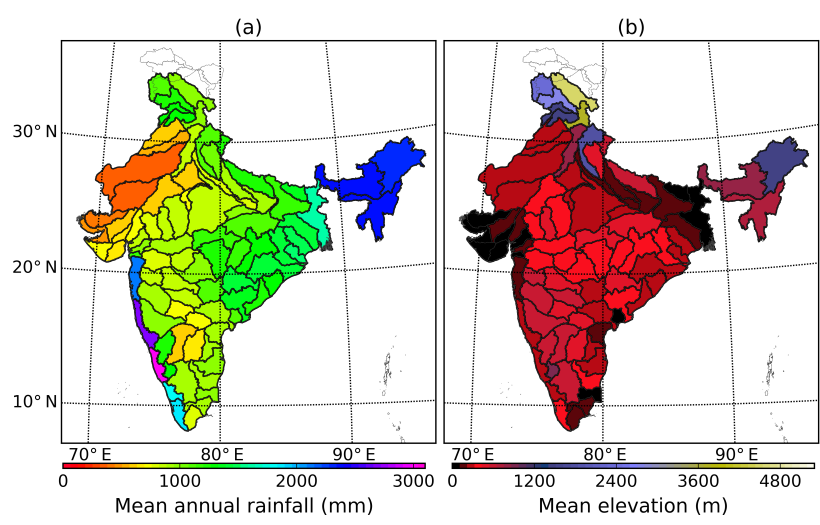

Figure 2. Spatial distributions of (a) long-term average annual rainfall (calculated from IMD gridded rainfall dataset during 19802010) and (b) average elevation above mean sea level (calculated using SRTM DEM) over 86 delineated river basins across India.

Western Ghats (located on the Indian west coast) and the northeastern basins receive the highest rainfall, with magnitudes going up to $3000 \mathrm{~mm}_{\text {year }}{ }^{-1}$. The Western Ghats receive orographic rainfall due to steep topographic gradient that exist from the west to the east, making the eastern part a leeward area where rainfall is mainly associated with the passage of lows and depressions developed in the Bay of Bengal (Prakash et al., 2016a). Details of the orographic features of rainfall over the Western Ghats can be found in Tawde and Singh (2015). The high rainfall in the northeastern part of India is associated with orographic control and multi-scale interactions of monsoon flow (Prakash et al., 2016a). Basins in the Indo-Gangetic plain and on the east coast receive above average rainfall of around $1400 \mathrm{~mm}_{\text {year }}{ }^{-1}$, governed by the tropical monsoons. The northwestern basins, associated with a semi-arid type of climate, receive low annual rainfall ranging from 300 to $400 \mathrm{~mm}_{\text {year }}{ }^{-1}$.

Figure $2 b$ shows the spatial distribution of the basinwise elevation above mean sea level (a.m.s.l.; details in Table S1). The northern tract of Jammu and Kashmir comprises the basins with highest elevations, between 2500 to $5000 \mathrm{~m}$ a.m.s.l. These basins suffer from scarce rain monitoring networks, due to which five of these high-elevation basins have been ignored in the analysis. High pitch mountains are also found in the northeastern basins where basin-wise elevation goes as high as $1400 \mathrm{~m}$ a.m.s.l. The Western Ghats are characterized by a sharp topographic gradient with the elevations increasing from around $200 \mathrm{~m}$ a.m.s.l. on the west coast to beyond $600 \mathrm{~m}$ a.m.s.l. as you move east. This transition results in heavy orographic rainfall on the west coast and leads to the sharp rainfall contrast on the leeward side of the Western Ghats.
Rainfall-runoff modeling was done on the Hirakud catchment of the Mahanadi River basin and the Wainganga catchment of the Godavari River basin. The Mahanadi River basin, situated near the eastern coast of India, is one of the largest Indian basins draining an area of $141000 \mathrm{~km}^{2}$. It is prone to frequent flooding in the downstream part, with five major flood events in the first decade of the 21 st century (Jena et al., 2014). In the upstream part of the Mahanadi River basin is a multi-purpose dam (Hirakud), which encompasses a catchment area of around $85200 \mathrm{~km}^{2}$ (Fig. 1b). Hirakud dam started its operations in 1957 and its upstream does not include any major dam, although a number of small-scale irrigation reservoirs are operational during the monsoon. Agricultural, forest and shrub land account for around 55, 35 and $7 \%$, respectively, of the total basin coverage (Kneis et al., 2014). The Wainganga river basin, the largest sub-basin of the Godavari basin (located in peninsular India), drains a total area of $51422 \mathrm{~km}^{2}$. Both basins receive an annual rainfall of around $1500 \mathrm{~mm}$.

\subsection{Datasets used}

The IMD gridded rainfall dataset was used as the reference product and TRMM and IMERG were compared against IMD. A brief summary of the datasets is given in Table 1 . A brief introduction to the three rainfall datasets is given below.

\subsubsection{Gridded IMD and streamflow dataset}

The IMD gridded precipitation dataset provides daily rainfall estimates over the Indian landmass from 1901 to 2014 at a spatial resolution of $0.25^{\circ} \times 0.25^{\circ}$. It has been developed using a dense network of rain gauges consisting of 6955 stations and is known to reasonably capture the heavy orographic rainfall in the Western Ghats and the northeast, and the low rainfall on the leeward side of the Western Ghats. Details about the number of stations used to make the gridded product are discussed in the Supplement. For a detailed discussion on the evolution of the IMD gridded dataset, refer to Pai et al. (2014).

It is to be noted that IMD measures rainfall accumulation at 08:30 IST ( Indian Standard time or 03:00 UTC). The accumulated rainfall for the previous day is provided as the rainfall estimate for the current day. For instance, the IMD rainfall estimate at a gauging station for 14 September 2014 refers to the rainfall accumulation from 08:30 IST (03:00 UTC) on 13 September 2014 to 08:30 IST (03:00 UTC) on 14 September 2014. Both TRMM and IMERG precipitation estimates were converted to IMD the timescale.

The gridded daily minimum and maximum temperature was obtained from IMD at a spatial resolution of $1^{\circ} \times 1^{\circ}$ (Srivastava et al., 2009). Daily wind speed data were obtained from coupled National Centers for Environmental Pre- 
Table 1. Summary of the precipitation datasets used.

\begin{tabular}{|c|c|c|c|c|c|}
\hline Product name & $\begin{array}{l}\text { Spatial } \\
\text { resolution }\end{array}$ & $\begin{array}{l}\text { Temporal } \\
\text { resolution }\end{array}$ & $\begin{array}{l}\text { Spatial } \\
\text { coverage }\end{array}$ & Temporal coverage & Period used in this study \\
\hline IMD gridded rainfall & $0.25^{\circ} \times 0.25^{\circ}$ & Daily & $\begin{array}{l}\text { Indian } \\
\text { landmass }\end{array}$ & 1901-2014 & $\begin{array}{l}\text { 1998-2013, } \\
12 \text { March-31 December } 2014\end{array}$ \\
\hline TRMM research product & $0.25^{\circ} \times 0.25^{\circ}$ & 3-hourly & $50^{\circ} \mathrm{N}-\mathrm{S}$ & 1998-present & $\begin{array}{l}\text { 1998-2013, } \\
12 \text { March-31 December } 2014\end{array}$ \\
\hline IMERG Final run & $0.1^{\circ} \times 0.1^{\circ}$ & Half-hourly & $60^{\circ} \mathrm{N}-\mathrm{S}$ & 12 March 2014-present & 12 March-31 December 2014 \\
\hline
\end{tabular}

diction (NCEP) and Climate Forecast System Reanalysis $(\mathrm{CFSR})$ at a spatial resolution of $0.5^{\circ} \times 0.5^{\circ}$. Daily discharge data at the inflow site of the Hirakud reservoir was obtained from the state Water Resources Department of Odisha and the Hirakud Dam Project, Burla, Sambalpur. Daily discharge data at the Wainganga basin was obtained through the WRIS website (http://www.india-wris.nrsc.gov.in/wris.html).

\subsubsection{Tropical Rainfall Measuring Mission (TRMM)}

In order to provide a high-resolution precipitation dataset in real-time, the TRMM satellite was launched in late 1997 and it provides 3-hourly rainfall estimates from 1998 to the current date at a quasi-global coverage $\left(50^{\circ} \mathrm{N}-\mathrm{S}\right)$ at a spatial resolution of $0.25^{\circ} \times 0.25^{\circ}$ (Huffman et al., 2007). Two variants of TMPA are available: a real-time product, which is available at 3-6h latency, and the research product, which is available at 2-months latency. The TRMM research product makes use of rain gauge stations from the Global Precipitation Climatology Centre (GPCC) to post-process the TRMM estimates, details of which can be found in Huffman et al. (2007). We used the TRMM research product in this study (henceforth mentioned as TRMM).

\subsubsection{Integrated Multi-satellitE Retrievals for GPM (IMERG)}

IMERG is the day-1 multi-satellite precipitation algorithm for GPM which combines data from TMPA, PERSIANN, CMORPH and NASA PPS (Precipitation Processing System). For a detailed understanding of the retrieval algorithm of IMERG, refer to Huffman et al. (2015) and Liu (2016).

The major advancement in the GPM satellite is the improved sensitivity of sensors leading to improved detection of low precipitation rates $\left(<0.5 \mathrm{~mm} \mathrm{~h}^{-1}\right)$ and falling snow, a known shortcoming of TRMM. IMERG is available in three variants, (a) Early run (latency $\sim 6 \mathrm{~h}$ ), (b) Late run (latency $\sim 18 \mathrm{~h}$ ) and (c) Final run (latency $\sim 4$ months) (Liu, 2016). Each product is available at half-hourly temporal and $0.1^{\circ} \times 0.1^{\circ}$ spatial resolutions. The spatial coverage is $60^{\circ} \mathrm{N}-\mathrm{S}$ which is planned to be extended to $90^{\circ} \mathrm{N}-\mathrm{S}$ in the near future. We used the Final run product in our analysis.
Table 2. Contingency table used to calculate probability of detection (POD) and false alarm ratio (FAR) at a given rainfall threshold.

\begin{tabular}{lll}
\hline & \multicolumn{2}{c}{ Simulated } \\
\cline { 3 - 3 } & $>$ Threshold $\leq$ Threshold \\
\hline$\vec{D}$ & & \\
$\vec{D}_{0}^{0} \leq$ Threshold & HIT & MISS \\
$\overrightarrow{0}^{\circ} \leq$ Threshold & FALSE & NEGATIVE \\
\hline
\end{tabular}

\subsection{VIC hydrological model}

VIC is a macroscale semi-distributed hydrological model which uses a grid-based approach to quantify different hydro-meteorological processes by solving water balance and energy flux equations, specifically designed to represent the surface energy and hydrologic fluxes at varying scales (Liang et al., 1994, 1996). VIC uses multiple soil layers with variable infiltration, non-linear baseflow and addresses the sub-grid scale variability in vegetation. A stand-alone routing model (Lohmann et al., 1996) is used to generate runoff and baseflow at the outlet of each grid cell, assuming linear and time-invariant runoff transport. The land surface parameterization of VIC is coupled with a routing scheme in which the drainage system is conceptualized by connectedstem rivers at a grid scale. The routing model extends the FDTF-ERUHDIT (First Differenced Transfer FunctionExcess Rainfall and Unit Hydrograph by a Deconvolution Iterative Technique) approach (Duband et al., 1993) with a timescale separation and liberalized Saint-Venant equation type river routing model. The model assumes the runoff transport process to be linear, stable and time invariant.

VIC has been successfully used in a number of global and local hydrologic studies (Hamlet and Lettenmaier, 1999; Shah and Mishra, 2016; Tong et al., 2014; Wu et al., 2014; Yong et al., 2012). A recent commentary on the need for process-based evaluation of large-scale hyper-resolution models by Melsen et al. (2016) provides interesting insights into the use of VIC at different spatial scales and why we should not just decrease the grid size (hence increasing the spatial resolution of the model) without considering the dom- 
Table 3. Summary of different statistical indices used to evaluate the satellite precipitation products.

\begin{tabular}{llcc}
\hline Index & Formula & Best value & Worst value \\
\hline Pearson correlation $(R)$ & $\frac{\sum(X-\bar{X})(Y-\bar{Y})}{\sqrt{\sum(X-\bar{X})^{2}} \sqrt{\sum(Y-\bar{Y})^{2}}}$ & 1 & 0 \\
\hline Percentage bias (P-bias) & $\frac{\sum(Y-X)}{\sum X} \times 100$ & 0 & $+\infty /-\infty$ \\
\hline Probability of detection (POD) & $\frac{\text { HIT }}{\text { HIT }+ \text { MISS }}$ & 1 & 1 \\
\hline False alarm ratio (FAR) & $\frac{\text { FALSE }}{\text { HIT }+ \text { FALSE }}$ & 0 & $-\infty$ \\
\hline Nash-Sutcliffe efficiency (NSE) & $1-\frac{\sum(X-Y)^{2}}{\sum(X-\bar{X})^{2}}$ & 1 & 1 \\
\hline Root mean square error (RMSE) & $\sqrt{\frac{\sum(X-Y)^{2}}{n}}$ & & $\begin{array}{c}\text { (negative value means that mean is } \\
\text { a better estimator than the model). }\end{array}$ \\
\hline
\end{tabular}

$X=$ observed, $\bar{X}=$ observed mean, $Y=$ simulated, $\bar{Y}=$ simulated mean and $n=$ data points.

Table 4. Segregation of overall rainfall time series into low, medium and high rainfall time series (RF is rainfall, $\mu$ is mean of rainfall, and $\sigma$ is standard deviation of rainfall).

\begin{tabular}{ll}
\hline Rainfall regime & Criterion \\
\hline Low & $\mathrm{RF}<\mu$ \\
Medium & $\mathrm{RF} \geq \mu$ and $\mathrm{RF} \leq \mu+2 \sigma$ \\
High & $\mathrm{RF}>\mu+2 \sigma$ \\
\hline
\end{tabular}

inant processes at that scale. In line with the discussions in Melsen et al. (2016), VIC was run at a grid size of $0.5^{\circ} \times 0.5^{\circ}$ for the Hirakud basin and at $0.25^{\circ} \times 0.25^{\circ}$ for the Wainganga basin.

\subsection{Methodology}

All the analyses were performed at the basin scale. Basinwise daily mean areal rainfall was calculated for all three rainfall products (IMD, TRMM and IMERG) using the Thiessen Polygon method (Schumann, 1998) for their respective periods of availability.

In order to statistically evaluate the precipitation products, two skill measures were used (Pearson correlation coefficient, $R$, and percentage bias, $P$-bias) along with two threshold statistics (probability of detection, POD, and false alarm ratio, FAR). Table 2 shows the contingency table and Table 3 provides a summary of the statistical indices.

All the statistical inferences were drawn for the overall time series, and then separately for the different rainfall regimes. Table 4 shows the criterion to segregate the rainfall time series into different components. For computing POD and FAR for a different rainfall regime, a threshold is re- quired. The 25 th percentile value was selected as the threshold for the low rainfall regime, 50th percentile for medium regime, 75 th percentile for the high rainfall regime and 95th percentile for the very high rainfall regime. The statistical indices were calculated basin-wise.

In order to identify systematic bias in the satellite products, one meteorologic index (long-term basin mean annual rainfall) and one topographic index (basin mean elevation) was computed for the 86 basins. The long-term mean annual rainfall was computed using an IMD gridded dataset from 1980 to 2010 (31 years). Basin mean digital elevation model (DEM) was extracted from the Shuttle Radar Topography Mission (SRTM) DEM, and mean elevation was obtained on a basin-wise scale.

Due to the limited availability of IMERG data (starting from 2014), calibration of VIC was done using an approach similar to the one used by Tang et al. (2016b). First, VIC was calibrated (2000-2011) and validated (2011-2014) using gridded IMD precipitation time series. VIC was then calibrated (2000-2011) and validated (2011-2014) with TRMM precipitation time series. Further, both the IMD and TRMM calibrated models were validated with IMERG and TRMM for the year 2014 (from 1 April to 31 December 2014). The year 2000 was used as a warm up period for the model.

In line with the recent discussion by McCuen (2016) on the correct usage of statistical and graphical indices to evaluate model calibration and validation, four statistical parameters (Nash-Sutcliffe efficiency, NSE; percentage bias, $P$-bias; coefficient of determination, $R^{2}$; and root mean square error, RMSE) were used to evaluate the runoff simulations from VIC. Table 3 provides a summary of these indices. 


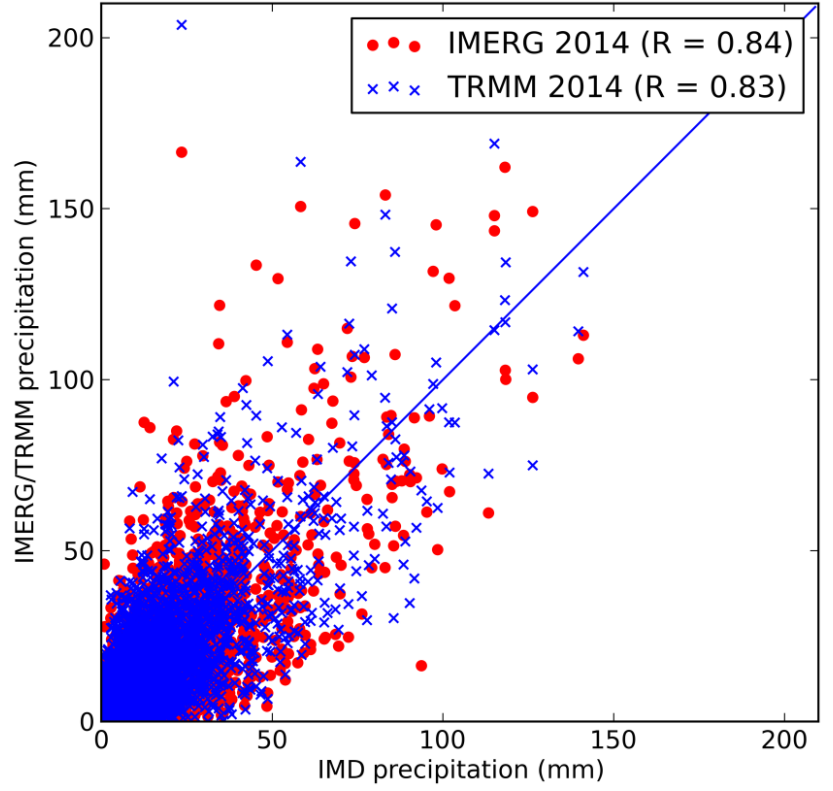

Figure 3. Scatter plot of satellite precipitation products (TRMM and IMERG) vs. observed rainfall (IMD) computed over 86 delineated river basins across India (based on daily precipitation data from 12 March to 31 December 2014).

\section{Results}

All the TRMM statistics were obtained for two distinct periods (1998-2013 and 2014). For the year 2014, the IMERG precipitation estimates were available from 12 March 2014. Therefore, the TRMM statistics for the year 2014 were obtained from 12 March to 31 December 2014. Henceforth, for the sake of convenience, statistics of TRMM-R refer to the time period 1998-2013 and statistics of TRMM and IMERG refer to the time period 12 March to 31 December 2014.

\subsection{Scatter plots}

Figure 3 shows the scatter plot of IMERG and TRMM with respect to IMD precipitation combining data from all the 86 basins for the year 2014. IMERG shows better correlation in 60 out of 86 basins. On looking at the scatter plots for individual basins (Fig. 4), IMERG tends to be better correlated to IMD than TRMM. It can be seen that the correlation values go as high as 0.96 for IMERG (and 0.94 for TRMM) with a very uniform spread across the $1: 1$ line for the five best basins (Fig. 4a-e; decided on the basis of correlation of IMERG with IMD in 2014). These basins are situated in the flat Deccan Plateau belt in south-central India (mostly concentrated in the Tapi and Godavari basins). For the other five basins (Fig. $4 \mathrm{f}-\mathrm{j}$ ), the poor correlation is due to the gross overestimation of IMERG/TRMM over IMD. Four of these five basins are situated in the high-elevation basins in northern India, which hints at a systematic dependence of
Table 5. Comparison of the IMERG and TRMM based on the number of basins in which the satellite products show higher/lower correlation based on the year 2014 ( $R=$ Pearson correlation coefficient).

\begin{tabular}{lrr}
\hline Expression & IMERG & TRMM \\
\hline$R>0.8$ & 73 & 68 \\
$R>0.9$ & 20 & 13 \\
Higher $R$ & 60 & 26 \\
Higher $R$ (low rainfall regime) & 52 & 34 \\
Higher $R$ (medium rainfall regime) & 52 & 34 \\
Higher $R$ (high rainfall regime) & 55 & 31 \\
\hline
\end{tabular}

IMERG/TRMM estimates with elevation. This is explored in detail in Sect. 3.5.

\subsection{Basin-wise correlation}

A basin-wise correlation was computed for retrospective analysis of TRMM-R and to compare TRMM and IMERG rainfall estimates for the year 2014. Table 5 provides a summary of the number of basins where IMERG/TRMM has a higher correlation. IMERG gives better rainfall estimates in the majority of basins for all rainfall regimes. The decomposition of the overall time series into different rainfall regimes reduces the correlation, which can be attributed to temporal smoothening in longer time series.

The spatial maps (Fig. 5) provide an illustration of the slight improvement of IMERG over TRMM with spatially coherent patterns. In the overall spatial maps (Fig. 5b-c), for the year 2014, TRMM and IMERG show similar skill, with IMERG capturing the rainfall slightly better in central and southern India. Both show similar skill in the high rainfall areas of the Western Ghats and the northeastern basins. IMERG gives slightly better estimates in the high-elevation basins in north India. There is no significant improvement in the basins located on the eastern coast (like the Mahanadi river basin). TRMM provides slightly better estimates of rainfall in the semi-arid basins located in the northwestern part of India. It is to be noted that TRMM statistics for 2014 are much better than its retrospective statistics (TRMM-R) with spatial coherent trends.

The low rainfall estimates (Fig. $5 \mathrm{~d}-\mathrm{f}$ ) over the semi-arid northwestern basins are slightly better for TRMM compared to IMERG. IMERG captures low rainfall better over the Indo-Gangetic plain. Both IMERG and TRMM show similar trends over the Western Ghats, northeastern basins, eastern coast and over the Deccan Plateau. IMERG does not capture the low rainfall regime over the Upper Indus basin (in northern India) and over the Upper Bhima and the Upper Godavari basins (in the Deccan Plateau belt).

The medium rainfall estimates (Fig. 5g-i) are best represented in central India and over the Deccan Plateau by TRMM and IMERG. Both show similar statistics over the 
(a) Basin ID: 78

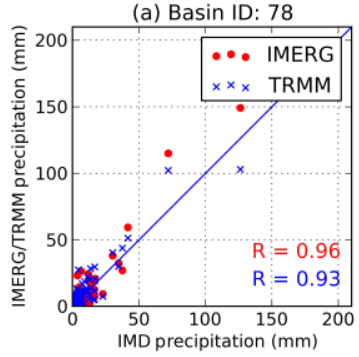

(f) Basin ID: 38

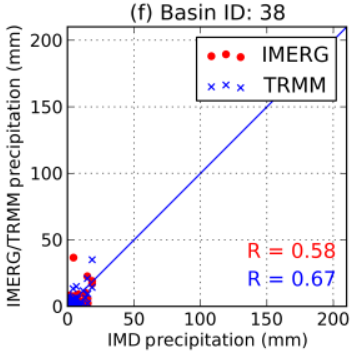

(b) Basin ID: 64

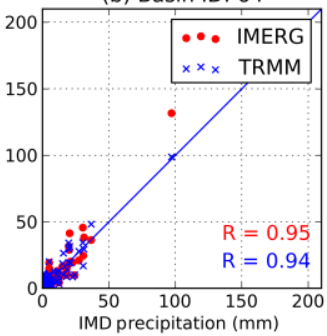

(g) Basin ID: 71

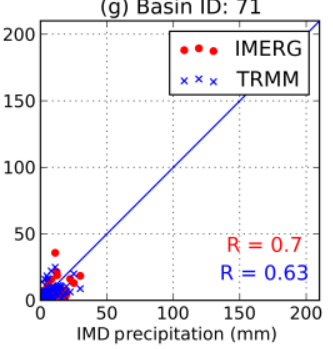

(c) Basin ID: 77

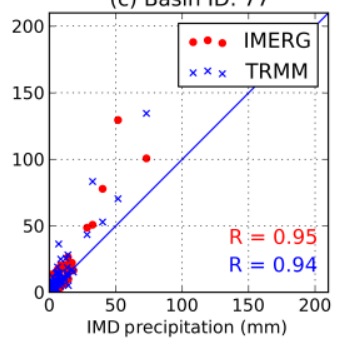

(h) Basin ID: 9

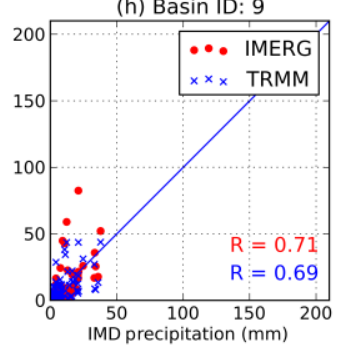

(d) Basin ID: 65

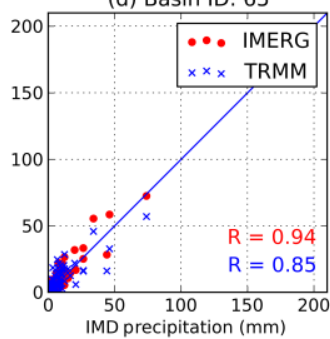

(i) Basin ID: 34

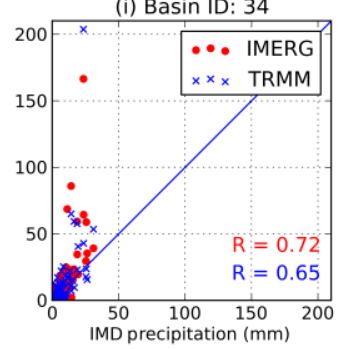

(e) Basin ID: 36

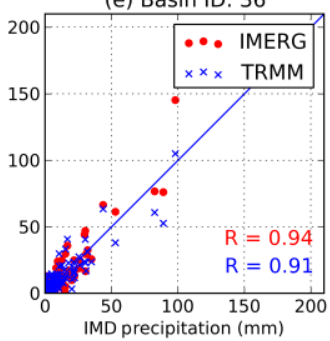

(j) Basin ID: 91

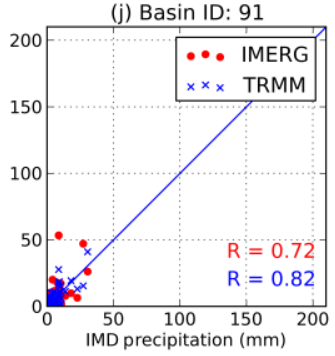

Figure 4. Scatter plot of satellite precipitation products (TRMM and IMERG) vs. observed rainfall (IMD) for the five best basins (a-e) in terms of correlation of IMERG with IMD (arranged in descending order) and the five worst basins (f-j) in terms of correlation of IMERG with IMD (arranged in ascending order) (based on daily precipitation data from 12 March to 31 December 2014).

Western Ghats and basins along the northeastern and eastern coast of India. TRMM slightly outperforms IMERG in the northwestern basin of Rajasthan, a trend also found in the low rainfall regime. IMERG does not capture the medium rainfall trends over the Upper Indus basin (in northern India). In general, TRMM-R medium rainfall estimates are best correlated in the semi-arid region of Rajasthan (northwestern basins) and in central India. There is not much variability in the correlation of medium rainfall trends of TRMM-R, with correlation coefficient mostly around 0.5 for the entire of India, except for the high-elevation Upper Indus basin.

The high rainfall estimates (Fig. 5j-k) show highest correlation in the Deccan Plateau belt, higher elevation basins in northern India, the Western Ghats and the east coast basins (except for the southernmost basin) for TRMM and IMERG. High rainfall estimates of TRMM are better correlated than IMERG in the northeastern basins of Brahmaputra and Barak and the northwestern basins of Rajasthan. Both show similar correlation over the high-elevation basins in the north and over the Western Ghats. IMERG outperforms TRMM in the rain-shadow area of the Western Ghats and in the southeastern basins of Pennar and Cauvery. Retrospective maps of TRMM-R (Fig. 5j) suggest that high rainfall is adequately captured in the Indo-Gangetic plain, Western Ghats, northwestern basins of Rajasthan, southeastern basins of Pennar and Cauvery and the eastern coast basins of central India. However, TRMM gives very low correlation values for the rain-shadow belt of the Western Ghats, suggesting that it does not capture the steep orographic gradient. The high rainfall estimates of TRMM-R give modest correlation in the north- eastern basins, high-elevation basins in northern India and the westernmost basins of the south (Varrar and Periyar).

\subsection{Basin-wise bias}

The basin-wise bias was computed for retrospective analysis of TRMM-R and to compare TRMM and IMERG rainfall estimates for the year 2014. Bias for the low rainfall regime (Fig. S2b) suggests that TRMM is more positively biased than IMERG for 75 out of 86 basins implying overestimation, which is a known problem with TRMM as its sensors cannot detect very low rainfall magnitudes $\left(<0.5 \mathrm{~mm} \mathrm{~h}^{-1}\right.$; Hou et al., 2014). If it detects a low intensity storm, it is most likely to overestimate (Fig. S2b). This seems to have improved in the IMERG product, due to the sensor improvements in the GPM mission (Huffman et al., 2015). The number of unbiased basins $(-10 \% \leq$ bias $\leq 10 \%)$ increased from 28 in TRMM to 37 in IMERG basins.

The spatial maps for the overall rainfall time series (Fig. 6a-c) suggest similar bias patterns in TRMM and IMERG with spatially coherent trends throughout most of India. IMERG gives a slightly smaller bias (closer to zero) over the high-elevation basins of north India (Upper Indus basin) and slightly larger bias (more negative) over the northeastern basins (of Brahmaputra and Barak) and the west flowing rivers of Kutch on the western coast in the state of $\mathrm{Gu}-$ jarat. IMERG and TRMM give large positive biases (overprediction) over the Upper and Middle Godavari basin (in the Deccan Plateau belt), which suggests that the sharp topographic gradient is not well captured. Retrospective maps of TRMM-R suggest underestimation over high-elevation basins in northern India (Indus, Jhelum and Chenab basins). 

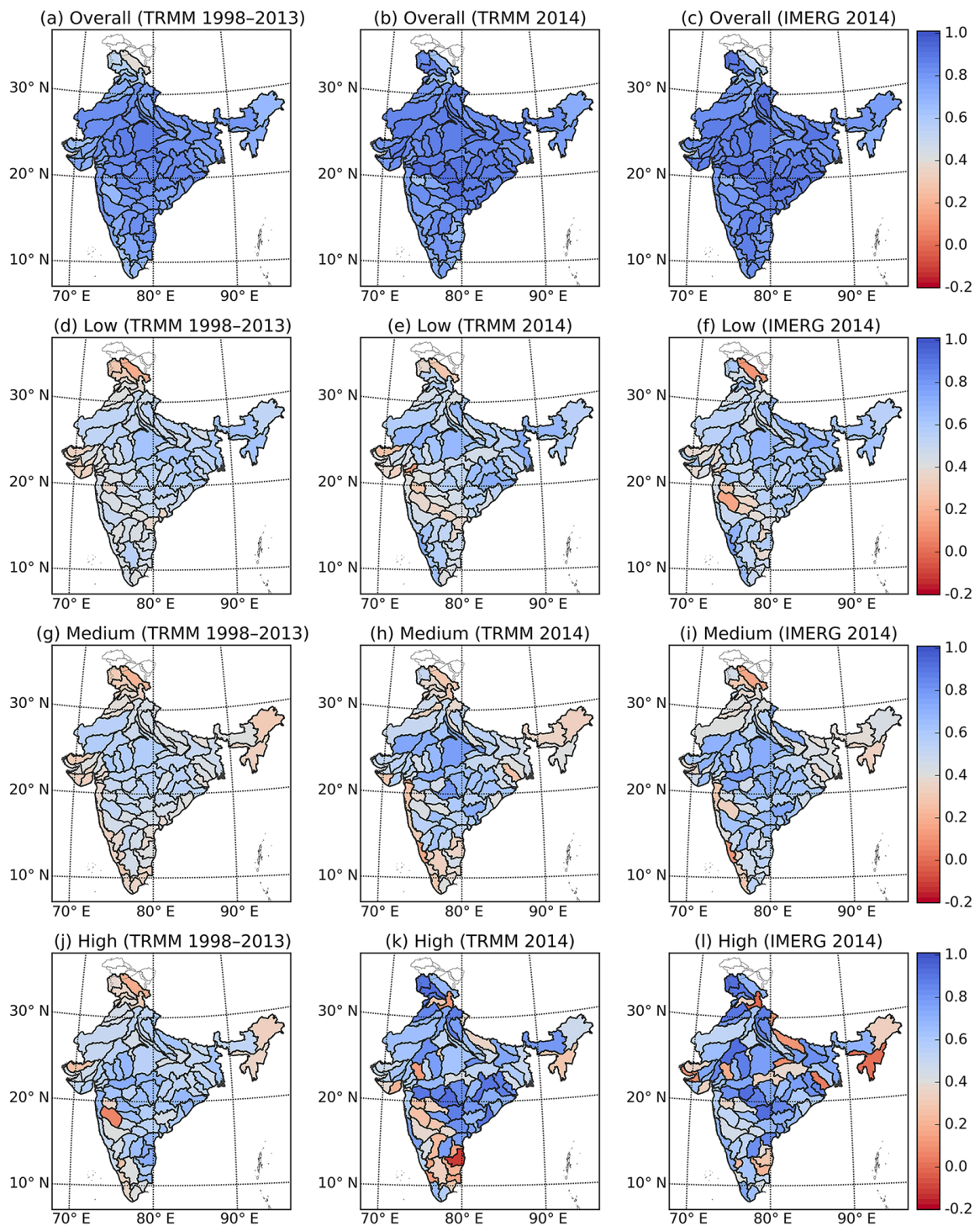

Figure 5. Spatial representation of the correlations of TRMM 1998-2013, TRMM 2014 and IMERG 2014 over 86 delineated river basins across India for $(\mathbf{a}-\mathbf{c})$ overall time series, $(\mathbf{d}-\mathbf{f})$ low, $(\mathbf{g}-\mathbf{i})$ medium and $(\mathbf{j}-\mathbf{l})$ high rainfall regimes.

However, TRMM captures the heavy precipitation on the Western Ghats well with low biases.

The low rainfall spatial maps (Fig. 6d-f) show large overprediction (positive bias) by TRMM (1998-2013 and 2014), which is improved in IMERG. The improvement is most prominent in the northeastern basins (Brahmaputra and Barak), central India (Mahi, Chambal and the Indo-Gangetic plain), the rain-shadow area of the Western Ghats and the southeastern coast. IMERG shows gross overprediction over the Luni basin (northwestern part of India). Retrospective TRMM-R maps for the low rainfall regime (Fig. 6d) show that the low rainfall was best captured in high rainfall areas of the Western Ghats, the Indo-Gangetic plain and the eastern coastal basins, which is not very surprising as TRMM does not detect low rainfall magnitudes very well, thus suffering from overprediction in arid and semi-arid basins. Improvement in the low rainfall sensors in IMERG has improved low rainfall estimates, but it still suffers from gross overprediction in semi-arid areas (as evident in the semi-arid basins in northwest India; Fig. 6f).

The medium rainfall spatial maps (Fig. 6g-i) suggest a similar spatial bias pattern in TRMM and IMERG. Both TRMM and IMERG suffer from underprediction (negative bias) in the high-elevation northern basins (of Indus and Jhelum), although IMERG seem to be less biased than TRMM. Both show similar trends in the Western Ghats, with low bias. However, both the products show large positive bias (overprediction) in the Middle Godavari basin, un- 

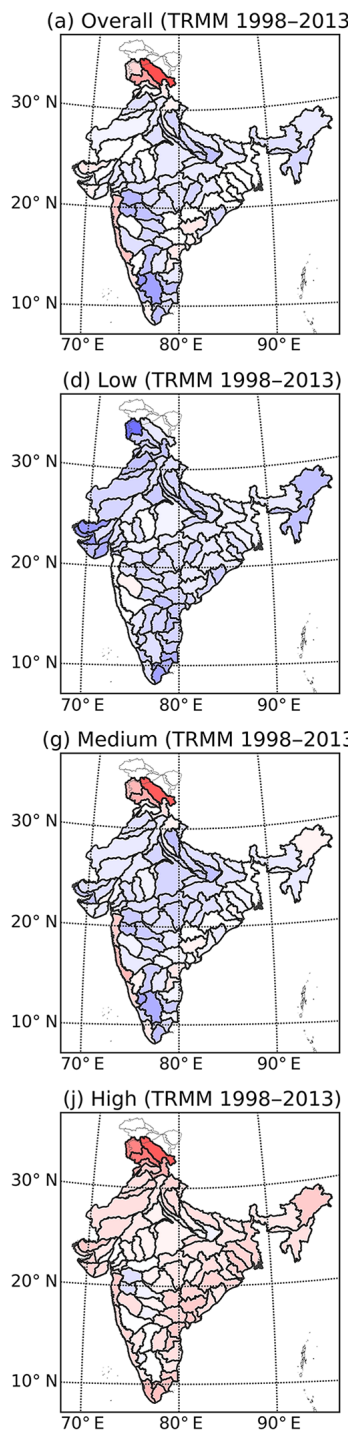
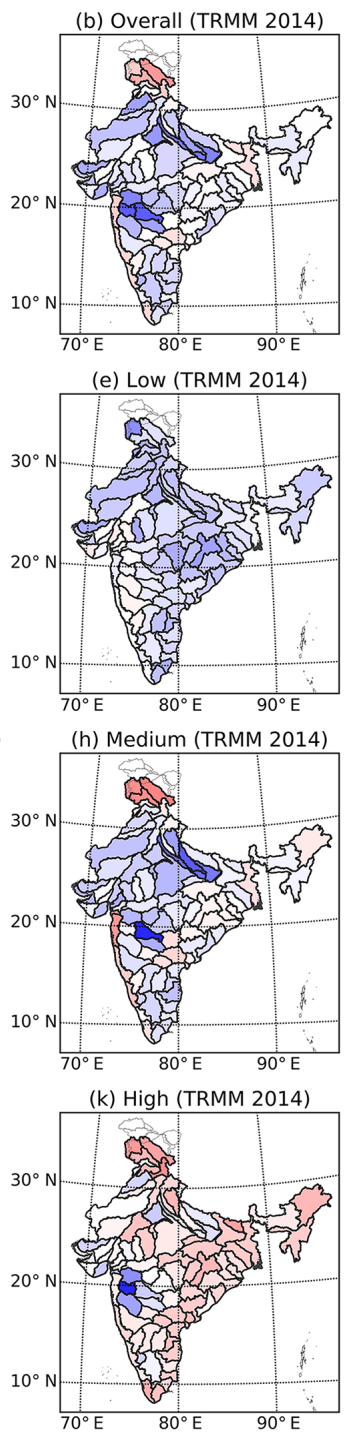
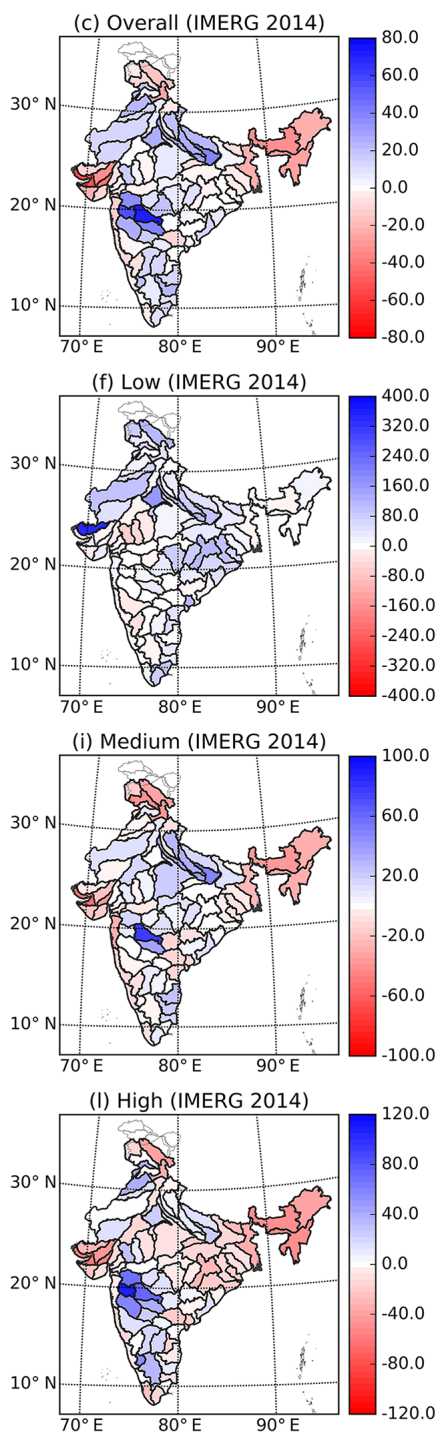

Figure 6. Spatial representation of percentage bias of TRMM 1998-2013, TRMM 2014 and IMERG 2014 over 86 delineated river basins across India for $(\mathbf{a}-\mathbf{c})$ overall time series and over $(\mathbf{d}-\mathbf{f})$ low, $(\mathbf{g}-\mathbf{i})$ medium and $(\mathbf{j}-\mathbf{l})$ high rainfall regimes.

able to capture the sharp topographic gradient in the region. IMERG slightly overpredicts rainfall in the northeastern basins (Brahmaputra and Barak). The retrospective TRMM maps for medium rainfall (Fig. 6g) show low bias over the entire of India, except over the Western Ghats (slight underprediction) and high-elevation northern basins of Indus and Jhelum (strong underprediction).

The high rainfall spatial maps (Fig. 6j-1) suggest a similar spatial pattern in TRMM and IMERG, with a slight negative bias over the majority of the basins. The high rainfall in the Western Ghats is well represented in TRMM and IMERG; however, with strong overprediction in the leeward side of the Western Ghats, suggesting that IMERG is unable to capture the sharp topographic gradients. IMERG shows greater underprediction in the high rainfall areas of the northeast- ern basins than TRMM; however, giving better estimates in the high-elevation basins in northern India. Both IMERG and TRMM give a similar bias pattern in the Indo-Gangetic plain and the semi-arid areas of the northwest. The retrospective TRMM-R map of high rainfall (Fig. 6j) suggests spatially homogeneous trends throughout India. However, it suffers from gross underestimation in the high-elevation basins of northern India (Indus, Jhelum and Chenab). It is clearly observed that the high-elevation basins are an outlier in most of the analysis. A systematic dependence of bias with elevation may be an underlying trend, which is further explored in Sect. 3.5. 

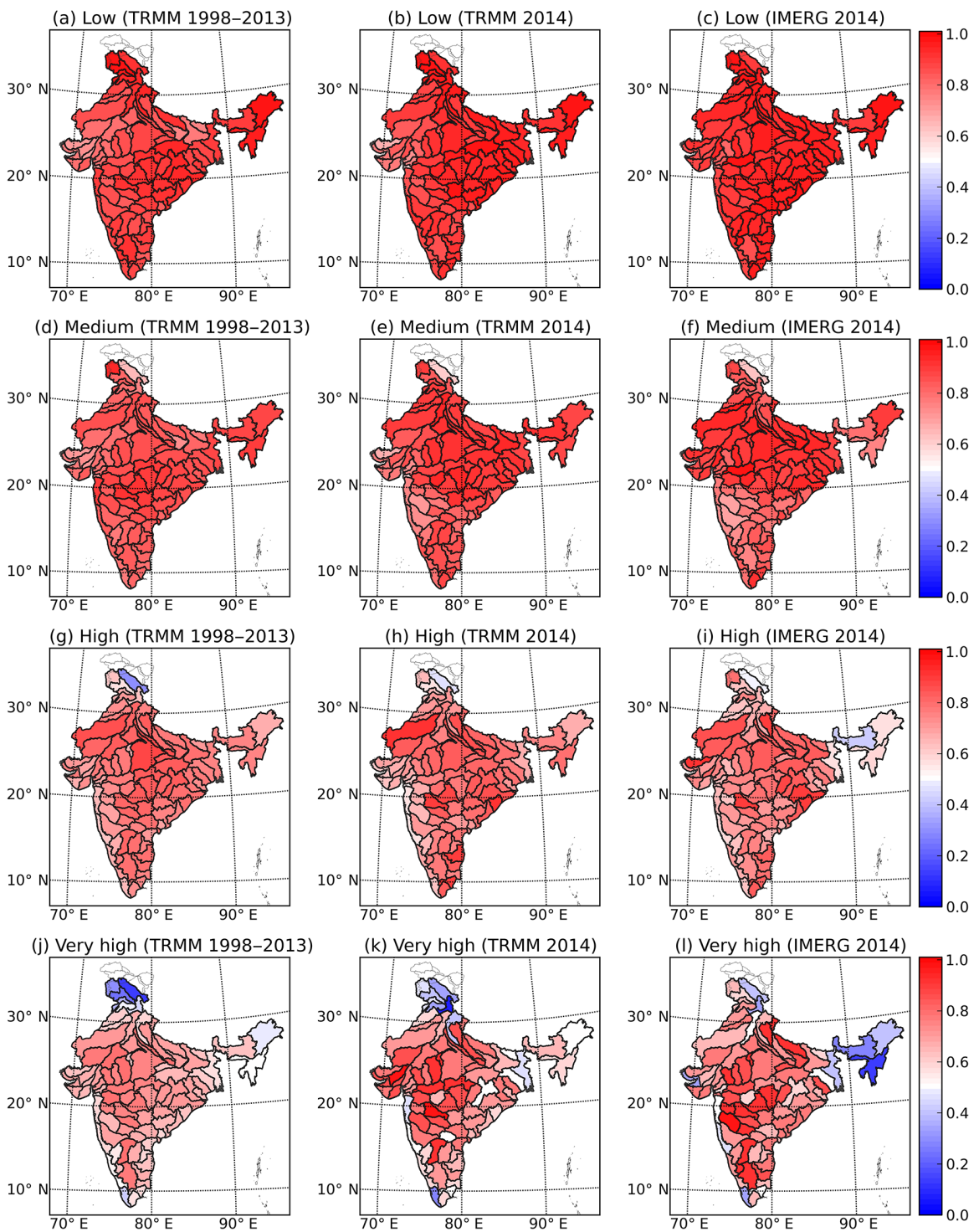

Figure 7. Spatial representation of probability of detection (POD) for (a-c) low (25 percentile), (d-f) medium (50 percentile), (g-i) high (75 percentile) and (j-l) very high (95 percentile) rainfall threshold for TRMM 1998-2013, TRMM 2014 and IMERG 2014 rainfall estimates over 86 delineated river basins across India.

Table 6. Comparison of IMERG and TRMM based on the number of basins in which the satellite products show higher/lower POD/FAR based on the year 2014. The last column gives the number of basins in which IMERG/TRMM gives similar POD/FAR. The low, medium, high and very high thresholds are the $25,50,75$ and 95 th percentile, respectively.

\begin{tabular}{lrrr}
\hline Expression & IMERG & TRMM & Similar \\
\hline Higher POD (low rainfall threshold) & 62 & 24 & 0 \\
Higher POD (medium rainfall threshold) & 39 & 37 & 10 \\
Higher POD (high rainfall threshold) & 32 & 45 & 9 \\
Higher POD (very high rainfall threshold) & 44 & 27 & 15 \\
Lower FAR (low rainfall threshold) & 42 & 40 & 4 \\
Lower FAR (medium rainfall threshold) & 53 & 26 & 7 \\
Lower FAR (high rainfall threshold) & 67 & 15 & 4 \\
Lower FAR (very high rainfall threshold) & 64 & 17 & 5 \\
\hline
\end{tabular}



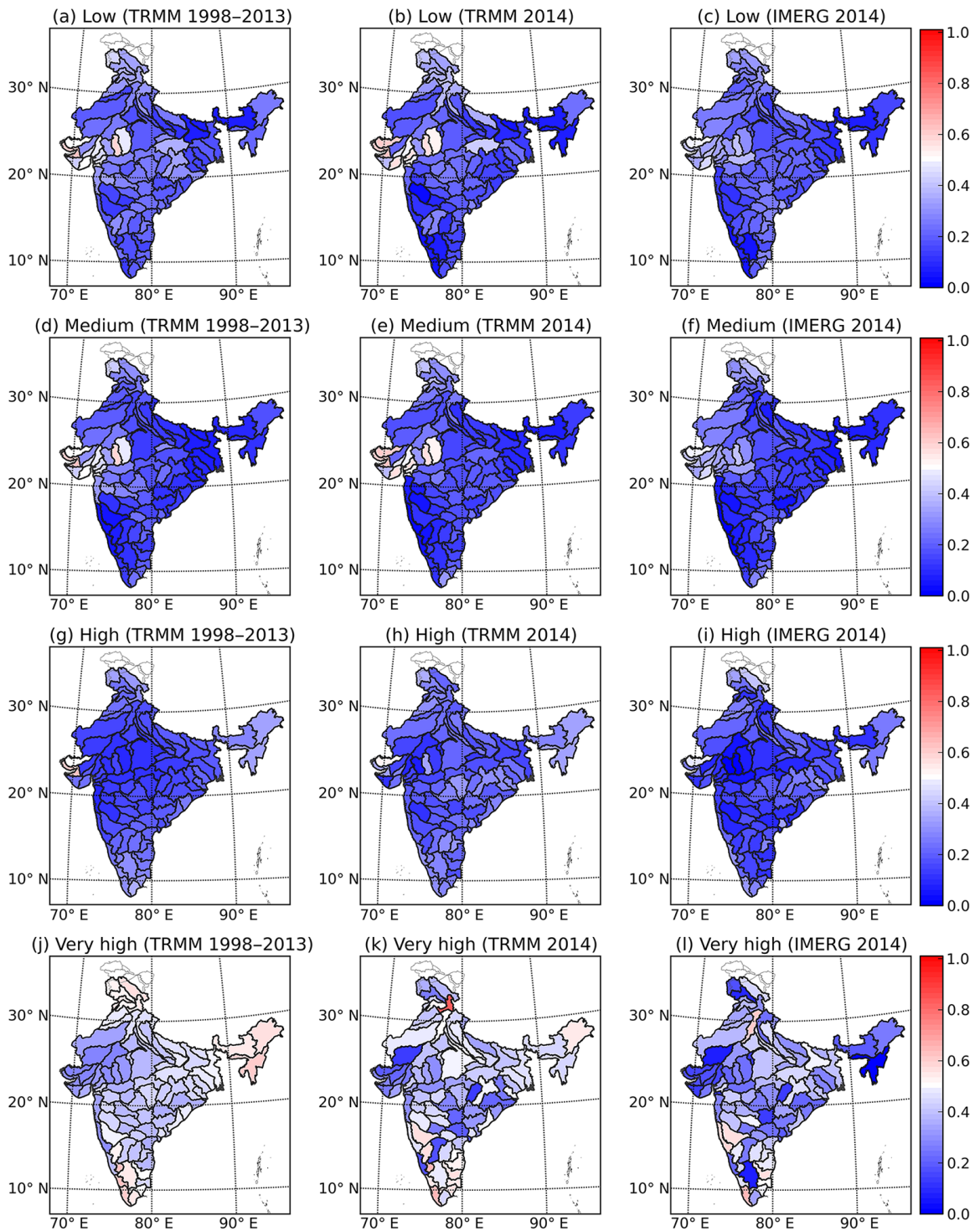

Figure 8. Spatial representation of false alarm ratio (FAR) for (a-c) low (25 percentile), (d-f) medium (50 percentile), (g-i) high (75 percentile) and (j-l) very high (95 percentile) rainfall threshold for TRMM 1998-2013, TRMM 2014 and IMERG 2014 rainfall estimates over 86 delineated river basins across India.

\subsection{Threshold statistics}

An increasing rainfall threshold leads to deteriorating trends in POD and FAR across the majority of the basins, with decreasing POD and increasing FAR. Table 6 summarizes the number of basins in which IMERG/TRMM gives higher/lower threshold statistics, including the basins in which they show similar results. At the low rainfall threshold, IMERG shows major improvement in POD in the western region of Gujarat (Luni, Bhadar and Setrunji basins; Fig. 7b, c). The average POD (low rainfall threshold) across basins is 0.95 for IMERG and 0.91 for TRMM. At the medium rainfall threshold, the average POD across basins is 0.87 for both
IMERG and TRMM. Notably, IMERG gives lower POD (medium rainfall threshold) in two (Barak and Brahmaputra lower sub-basin) out of the three northeastern basins, and higher POD (medium rainfall threshold) in the semi-arid basins of Rajasthan and Gujarat (Luni, Bhadar and Setrunji basins; Fig. 7e, f). At the high rainfall threshold, average POD across basins is 0.76 for IMERG and 0.77 for TRMM. There is a notable fall in performance in all three northwestern basins. IMERG gives slightly higher POD (high rainfall threshold) in the high-elevation northern basins (upper Indus and Jhelum basins; Fig. 7h, i). At the very high rainfall threshold, average POD across basins is 0.72 for IMERG and 0.7 for TRMM. At the very high rainfall threshold, it is 
clear that POD of IMERG is worse for all three northeastern basins and over the semi-arid basins of Rajasthan and $\mathrm{Gu}$ jarat (Fig. 7k, 1). There is a slight improvement in the POD values for the high-elevation northern basins (Chenab, Ravi, Beas and Satulaj basins).

At the low rainfall threshold, the average FAR across basins is 0.24 for TRMM and 0.22 for IMERG. At the medium rainfall threshold, average FAR across basins is 0.22 for TRMM and 0.19 for IMERG. Notably, IMERG outperforms TRMM at low and medium rainfall thresholds giving lower FARs in the western basins of Gujarat (Luni and Setrunji basins; Fig. 8b, c, e, f). At the high rainfall threshold, average FAR across basins is 0.18 for IMERG and 0.22 for TRMM. Slightly reduced FAR are seen in central India (Yamuna and Chambal basins) and the northeastern basins (Brahmaputra basin) in IMERG at the high rainfall threshold (Fig. 8h, i). At the very high rainfall threshold, average FAR across basins is 0.33 for IMERG and 0.41 for TRMM. There are notably fewer false alarms in IMERG estimates over the northern, northeastern basins and the Western Ghats at very high thresholds. Both products give similar FAR (very high threshold) along the eastern coast and Deccan Plateau basins.

POD for TRMM-R suggests decreasing POD and increasing FAR with increasing rainfall threshold (Figs. 7a, d, g, j and $8 \mathrm{a}, \mathrm{d}, \mathrm{g}, \mathrm{j}$ ). The average POD across basins is 0.89 , $0.85,0.77$ and 0.66 for low, medium, high and very high rainfall thresholds, respectively. The respective FAR values are $0.26,0.22,0.21$ and 0.43 . At the high and very high thresholds, POD drops significantly over the high-elevation northern basins and high rainfall northeastern basins and the Western Ghats (Fig. 7g, j). High FAR is recorded in the semi-arid basins in Gujarat and Rajasthan (Luni and Setrunji) and central India (Bhadar and Chambal) at low and medium rainfall thresholds (Fig. 8a, d) suggesting TRMM creates a lot of false alarms at low and medium rainfall magnitudes. There is a sharp contrast between FAR at high and very high thresholds, with low FAR at high rainfall threshold (75 percentile) and high FAR at very high threshold (95 percentile; Fig. $8 g, j$ ). This suggests that TRMM-R creates a lot of false alarms at very high rainfall thresholds, especially in the northeastern, northern and extreme southern basins (Fig. 8j).

\subsection{Systematic error in satellite estimates as a function of annual rainfall and mean elevation}

The satellite precipitation estimates were evaluated against a climatologic parameter (long-term annual rainfall of basin) and a topographic parameter (basin mean elevation) to investigate any systematic variation in errors with climatology or topography. We found there is no systematic dependence between the climatologic and topographic parameter ( $R=0.07$; Fig. S3) and they can be considered as independent (implying minimal interference).

TRMM-R rainfall estimates exhibited a very strong dependence on mean basin elevation, with decreasing skill (larger bias and lower correlation) in basins with high mean elevation (Figs. S4 and S5). For medium and high rainfall regimes (Fig. S4c, d), bias values were highly negative for high-elevation basins (especially for basins with mean elevation $>2000 \mathrm{~m}$ ), implying underprediction. The corresponding correlation values (Fig. S5c, d) also suggested reduced skill at high-elevation basins.

For the year 2014, the systematic dependence of bias on basin elevation improved in IMERG estimates, with correlation between basin-wise bias and elevation reducing from -0.43 to -0.32 for medium rainfall intensity (Fig. S6c) and from -0.31 to -0.08 for high rainfall intensity (Fig. S6d). The same was not observed in the correlation plots (Fig. S7). At low rainfall intensity (Fig. S7b), IMERG estimates exhibited a stronger systematic relationship between the basinwise correlation and elevation, with strongly decreasing correlation with elevation than TRMM. At medium rainfall intensity (Fig. S7c), both TRMM and IMERG showed decreasing skill with increasing elevation. This systematic dependence was stronger in IMERG than TRMM, as reflected in the higher negative correlation between basin-wise correlation and elevation in medium rainfall IMERG estimates (Fig. S7c).

The same analysis was repeated against mean annual precipitation (Figs. S8-S11 in the Supplement) wherein systematic error dependence was found to be smaller. TRMM-R rainfall estimates exhibited a systematic dependence of bias and correlation with basin-wise mean annual rainfall for low and medium rainfall estimates (Figs. S8 and S9). At low rainfall intensity, TRMM-R estimates for basins experiencing low annual rainfall were found to be strongly positively biased (Fig. S8b), implying significant over-estimation. For the year 2014, the systematic dependence of bias was reduced in IMERG at medium rainfall intensities (Fig. S10c, correlation improved from -0.43 in TRMM to -0.3 for IMERG). Substantial skill was lost in terms of decreasing correlation for basins receiving high rainfall in both TRMM and IMERG estimates (Fig. S11c). At high rainfall intensities, the bias was more negative (implying underprediction) in basins which received more rainfall in both IMERG and TRMM (Fig. S10d).

\subsection{Rainfall-runoff modeling}

Rainfall-runoff modeling was carried out over the Hirakud catchment of the Mahanadi River basin and the Wainganga catchment of the Godavari River basin, with the calibration and validation periods as 2000-2011 and 2012-2014, respectively. VIC was first calibrated with IMD gridded precipitation and then with TRMM 3B42V7. The two calibrated models were then forced with TRMM and IMERG precipitation for the year 2014 (April-December). Tables 7 and 8 show the model performances.

The IMD calibrated model showed better simulations compared to the TRMM calibrated model, with higher NSE, coefficient of determination and smaller bias and RMSE in 
Table 7. Performance statistics for rainfall-runoff modeling using VIC for the Hirakud catchment of the Mahanadi River basin.

\begin{tabular}{lrrrrr}
\hline & Time period & NSE & $R^{2}$ & $P$-bias & RMSE $\left(\mathrm{m}^{3} \mathrm{~s}^{-1}\right)$ \\
\hline IMD calibration & $2000-2011$ & 0.83 & 0.84 & 16.78 & 919.88 \\
IMD validation & $2012-2014$ & 0.86 & 0.88 & 3.91 & 823.58 \\
TRMM calibration & $2000-2011$ & 0.72 & 0.74 & 18.2 & 1160.94 \\
TRMM validation & $2012-2014$ & 0.73 & 0.74 & 14 & 1128.15 \\
TRMM (IMD calibration) & 2014 & 0.72 & 0.82 & -9.41 & 1591.09 \\
IMERG (IMD calibration) & 2014 & 0.64 & 0.68 & 41.4 & 1786.22 \\
TRMM (TRMM calibration) & 2014 & 0.72 & 0.82 & -9.24 & 1588.86 \\
IMERG (TRMM calibration) & 2014 & 0.7 & 0.72 & 31.32 & 1641.82 \\
\hline
\end{tabular}

Table 8. Performance statistics for rainfall-runoff modeling using VIC for Wainganga River basin.

\begin{tabular}{lrrrrr}
\hline & Time period & NSE & $R^{2}$ & $P$-bias & RMSE $\left(\mathrm{m}^{3} \mathrm{~s}^{-1}\right)$ \\
\hline IMD calibration & $2000-2011$ & 0.81 & 0.81 & 9.18 & 740.49 \\
IMD validation & $2012-2014$ & 0.87 & 0.88 & -10.8 & 852.9 \\
TRMM calibration & $2000-2011$ & 0.7 & 0.71 & 15.66 & 931.65 \\
TRMM validation & $2012-2014$ & 0.83 & 0.83 & 5.93 & 973.41 \\
TRMM (IMD calibration) & 2014 & 0.74 & 0.74 & 8.70 & 883.19 \\
IMERG (IMD calibration) & 2014 & 0.74 & 0.76 & -0.52 & 883.59 \\
TRMM (TRMM calibration) & 2014 & 0.72 & 0.75 & -2.70 & 922.04 \\
IMERG (TRMM calibration) & 2014 & 0.61 & 0.66 & -12.10 & 1082.34 \\
\hline
\end{tabular}

both the Wainganga and Hirakud basins. The TRMM calibrated model showed overprediction (positive bias) in the Hirakud basin, but was relatively unbiased in the Wainganga basin $(-10 \leq$ P-bias $\leq 10$; Tables 7 and 8$)$.

The IMERG simulations with IMD and TRMM calibrated models were slightly inferior in comparison with TRMM simulations for 2014 (NSE $=0.64$ for IMERG and 0.72 for TRMM in IMD calibration; NSE $=0.7$ for IMERG and 0.72 for TRMM in TRMM calibration) (Table 7 and Fig. 9) for Hirakud. However, the IMERG simulations gave similar results as TRMM in the Wainganga basin when calibrated using IMD data, but inferior results when calibrated with TRMM data $(\mathrm{NSE}=0.61$ for IMERG and 0.72 for TRMM) (Table 8 and Fig. 10). In the case of the Hirakud basin, IMERG simulations gave higher NSE when calibrated with TRMM data. However, in the case of the Wainganga basin, IMERG gave higher NSE when calibrated with IMD data. The high negative bias in IMERG simulations (with IMD and TRMM calibrated models) showed significant underprediction compared to TRMM.

Both TRMM and IMERG underestimated the magnitude of the two major peaks (flow $>15000 \mathrm{~m}^{3} \mathrm{~s}^{-1}$ ) in the Hirakud and Wainganga basins in 2014 (Figs. 9 and 10). However, the phase was well captured by both IMERG and TRMM in the two basins. IMERG overestimated low flows for the majority of time in both IMD and TRMM calibrated VIC model for both the basins, and thus was inferior in performance to TRMM. This suggests that the use of an appropriate post-processor for streamflow (Ye et al., 2014) could tremendously benefit the flow simulations, which might be an interesting study for the future.

\section{Conclusions}

TRMM 3B42 and IMERG precipitation estimates were comprehensively evaluated over 86 basins in India. TRMM 3B42 was analyzed for two distinct time periods, the retrospective analysis was carried out from 1998 to 2013 and the current estimates were compared with IMERG for the year 2014 (12 March-31 December 2014). The systematic biases in both estimates were explored with respect to a climatologic parameter (basin mean annual rainfall) and a topographic parameter (basin mean elevation). Finally, TRMM and IMERG were hydrologically evaluated by carrying out rainfall-runoff modeling over the Hirakud catchment of the Mahanadi River basin and the Wainganga catchment of the Godavari River basin. The results of the study are summarized as the following:

1. IMERG rainfall estimates were found to be better than TRMM at all rainfall intensities in terms of correlation. IMERG outperformed TRMM in 60, 52, 52 and 55 out of 86 basins for overall, low, medium and high rainfall regimes.

2. IMERG gave better estimates of low rainfall magnitudes with smaller biases in 75 out of the 86 basins analyzed, which suggests that the sensor improvement in 

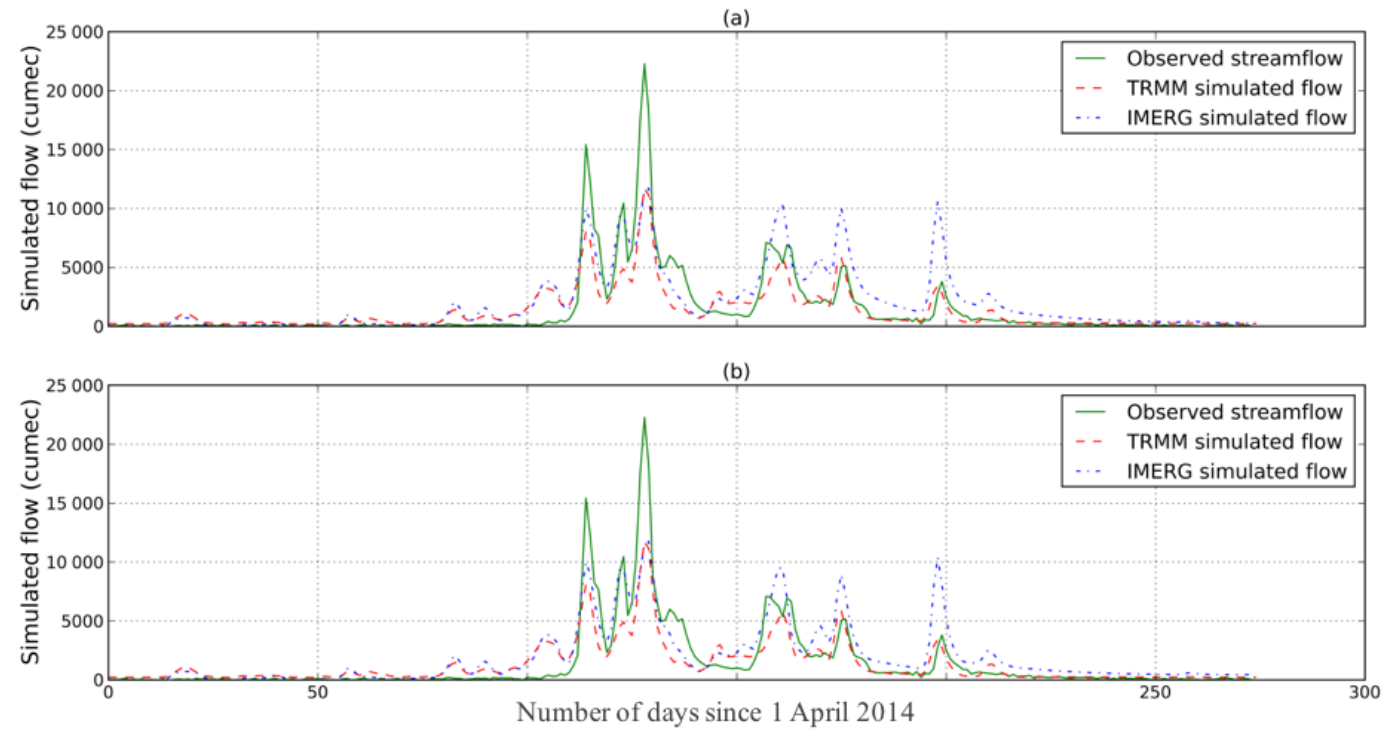

Figure 9. Hydrographs for TRMM and IMERG simulations (1 April-31 December 2014) with (a) IMD and (b) TRMM calibrated VIC model for the Hirakud basin.
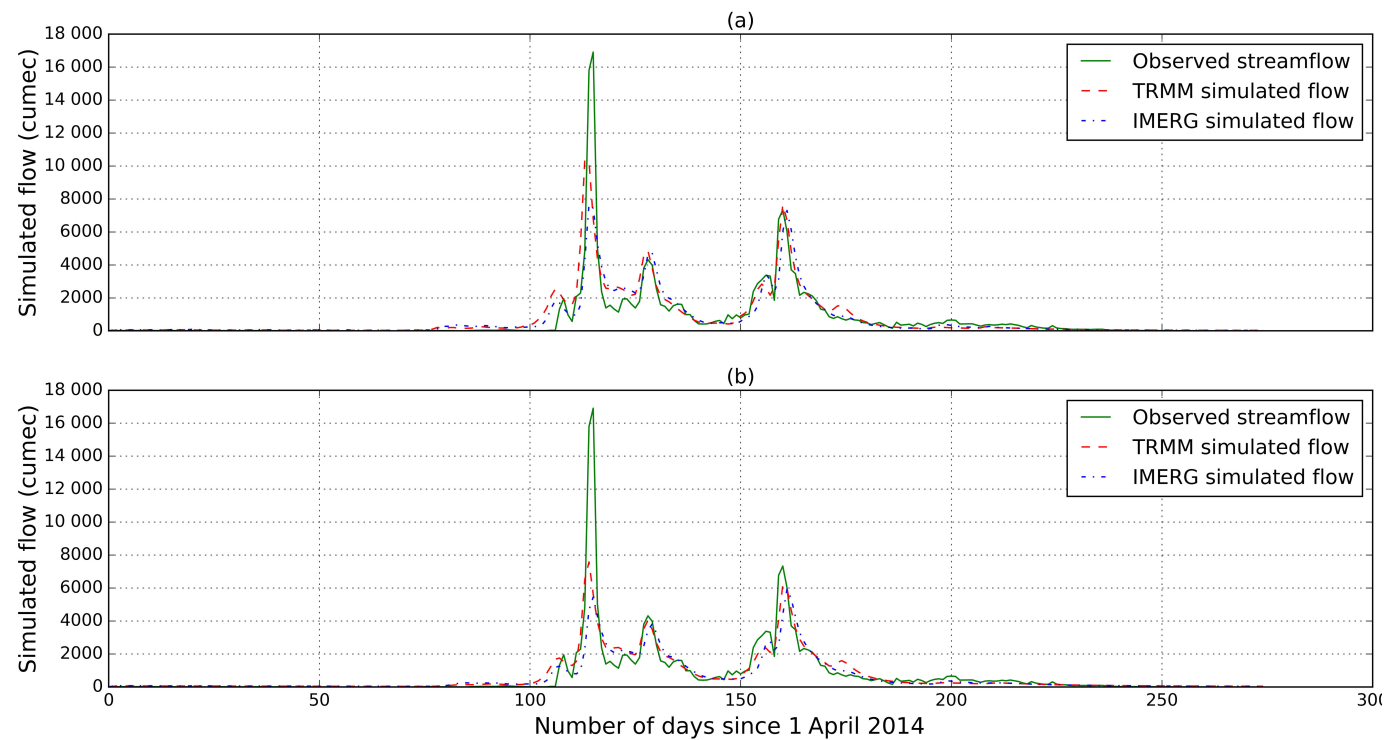

Figure 10. Hydrographs for TRMM and IMERG simulations (1 April-31 December 2014) with (a) IMD and (b) TRMM calibrated VIC model for the Wainganga basin.

IMERG satellite translated into better low rainfall estimation. IMERG captured the low rainfall magnitudes better over the Indo-Gangetic plain, northeastern basins of Brahmaputra and Barak, central India (Mahi and the Indo-Gangetic plain) and the rain-shadow area of the Western Ghats. However, for the semi-arid northwestern basins, TRMM low rainfall estimates outperformed IMERG.

3. The high rainfall estimates of IMERG outperformed TRMM in the rain-shadow area of the Western Ghats, the high-elevation basins of the north and the southeastern basins of Pennar and Cauvery. However, TRMM did a better job in the northeastern basins of Brahmaputra and Barak and the northwestern basins of Rajasthan.

4. Increasing rainfall thresholds leads to deteriorating trends in POD and FAR across the majority of basins, with decreasing POD and increasing FAR. At very high rainfall thresholds ( $>95$ percentile), TRMM exhibited high FAR, especially in the northeastern and southern basins, implying that they do not capture the extreme 
precipitation magnitudes well. This was also seen in the rainfall-runoff exercise where the peak flows were underpredicted in the Mahanadi and Wainganga River basins, both in the case of TRMM and IMERG.

5. The skill of TRMM-R medium rainfall estimates (in terms of P-bias and correlation) was found to exhibit strong systematic dependence on annual rainfall (climatologic parameter), with larger bias and lower correlation in basins which received higher annual rainfall. This systematic dependence was reduced significantly in IMERG estimates. However, no such improvement was found at low and high rainfall intensities.

6. A very strong deteriorating skill (increasing bias and decreasing correlation) was found in TRMM-R rainfall estimates at all intensities in the high-elevation basins. This systematic dependence was strongly reduced in IMERG estimates at all rainfall intensities, suggesting IMERG captures the rainfall trends better with respect to topography.

7. Rainfall-runoff modeling using the VIC model over the Mahanadi and Wainganga River basins gave better results with TRMM as input forcing rather than IMERG. Both TRMM and IMERG captured the phase of the peak flows, but both underreported the magnitudes. Low flows were grossly over predicted by IMERG, which led to overall poor performance with IMERG. As GPM is still a young mission, a longer time series of IMERG will help in model evaluation as IMERG can be used to directly calibrate the model, hence capturing the fine details in the product. It will also be useful to see if other hydrologic models can capture peak flows more accurately when forced with TRMM/IMERG in the Mahanadi and Wainganga basins. This would mean that the poor representation of peak flows is a function of model structural uncertainty, and not the satellite precipitation products driving the model. This will make a very interesting future case study.

In essence, IMERG gives reasonable improvement in rainfall estimates across the majority of the Indian basins. The most notable improvement in IMERG is the reduction in systematic error dependence on topography (basin mean elevation), which suggests improvements in the assimilation of satellite observations. The improved sensitivity of the $\mathrm{Ku}$ and Ka bands in the GPM satellite resulted in an improvement in the detection of low rainfall magnitudes. The expected improvement in IMERG in snow detection could not be verified in this study as India is mostly a tropical country which receives very scanty snowfall. The constant overestimation of low-flow magnitudes in the rainfall-runoff exercise suggest that IMERG may benefit from a post-forecast data assimilation scheme (or postprocessing; Ye et al., 2014), which is a worthy topic for further research.
Data availability. Rainfall data used in this study can be obtained from the India Meteorological Department (http://www.imd.gov. in). Streamflow data can be accessed through the India-WRIS portal (http://www.india-wris.nrsc.gov.in). The IMERG data can be obtained from the Precipitation Processing System (PPS), TRMM data from the Goddard Earth Sciences Data and Information Services Center (GES-DISC).

\section{The Supplement related to this article is available online at https://doi.org/10.5194/hess-21-6117-2017- supplement.}

Competing interests. The authors declare that they have no conflict of interest.

Edited by: Hannah Cloke

Reviewed by: two anonymous referees

\section{References}

Akhtar, M. K., Corzo, G. A., van Andel, S. J., and Jonoski, A.: River flow forecasting with artificial neural networks using satellite observed precipitation pre-processed with flow length and travel time information: case study of the Ganges river basin, Hydrol. Earth Syst. Sci., 13, 1607-1618, https://doi.org/10.5194/hess-131607-2009, 2009.

Artan, G., Gadain, H., Smith, J. L., Asante, K., Bandaragoda, C. J., and Verdin, J. P.: Adequacy of satellite derived rainfall data for stream flow modeling, Nat. Hazards, 43, 167-185, https://doi.org/10.1007/s11069-007-9121-6, 2007.

Bajracharya, S. R., Shrestha, M. S., and Shrestha, A. B.: Assessment of high-resolution satellite rainfall estimation products in a streamflow model for flood prediction in the Bagmati basin, Nepal, J. Flood Risk Manag., 10, 5-16, https://doi.org/10.1111/jfr3.12133, 2014.

Bisht, D. S., Chatterjee, C., Raghuwanshi, N. S., and Sridhar, V.: Spatio-temporal trends of rainfall across Indian river basins, Theor. Appl. Climatol., 1-18, https://doi.org/10.1007/s00704017-2095-8, online first, 2017.

Collischonn, B., Collischonn, W., and Tucci, C. E. M.: Daily hydrological modeling in the Amazon basin using TRMM rainfall estimates, J. Hydrol., 360, 207-216, https://doi.org/10.1016/j.jhydrol.2008.07.032, 2008.

Duband, D., Obled, C., and Rodriguez, J. Y.: Unit hydrograph revisited: an alternate iterative approach to $\mathrm{UH}$ and effective precipitation identification, J. Hydrol., 150, 115-149, https://doi.org/10.1016/0022-1694(93)90158-6, 1993.

Gao, Y. C. and Liu, M. F.: Evaluation of high-resolution satellite precipitation products using rain gauge observations over the Tibetan Plateau, Hydrol. Earth Syst. Sci., 17, 837-849, https://doi.org/10.5194/hess-17-837-2013, 2013.

Government of India: Watershed Atlas of India, available at: http: //india-wris.nrsc.gov.in/Publications/WatershedSubbasinAtlas/ WatershedAtlasofIndia.pdf, 2014.

Hamlet, A. F. and Lettenmaier, D. P.: Columbia River Streamflow Forecasting Based on ENSO and PDO Climate Signals, J. Wa- 
ter Res. Pl., 125, 333-341, https://doi.org/10.1061/(ASCE)07339496(1999)125:6(333), 1999.

Hou, A. Y., Kakar, R. K., Neeck, S., Azarbarzin, A. A., Kummerow, C. D., Kojima, M., Oki, R., Nakamura, K., and Iguchi, T.: The Global Precipitation Measurement Mission, B. Am. Meteorol. Soc., 95, 701-722, https://doi.org/10.1175/BAMS-D-1300164.1, 2014.

Huffman, G. J., Bolvin, D. T., Nelkin, E. J., Wolff, D. B., Adler, R. F., Gu, G., Hong, Y., Bowman, K. P., and Stocker, E. F.: The TRMM Multisatellite Precipitation Analysis (TMPA): Quasi-Global, Multiyear, Combined-Sensor Precipitation Estimates at Fine Scales, J. Hydrometeorol., 8, 38-55, https://doi.org/10.1175/JHM560.1, 2007.

Huffman, G. J., Bolvin, D. T., and Nelkin, E. J.: Integrated MultisatellitE Retrievals for GPM (IMERG) Technical Documentation, NASA/GSFC Code, 2015.

Jena, P. P., Chatterjee, C., Pradhan, G., and Mishra, A.: Are recent frequent high floods in Mahanadi basin in eastern India due to increase in extreme rainfalls?, J. Hydrol., 517, 847-862, https://doi.org/10.1016/j.jhydrol.2014.06.021, 2014.

Kneis, D., Chatterjee, C., and Singh, R.: Evaluation of TRMM rainfall estimates over a large Indian river basin (Mahanadi), Hydrol. Earth Syst. Sci., 18, 2493-2502, https://doi.org/10.5194/hess-182493-2014, 2014.

Liang, X., Lettenmaier, D. P., Wood, E. F., and Burges, S. J.: A simple hydrologically based model of land surface water and energy fluxes for general circulation models, J. Geophys. Res., 99, 14415, https://doi.org/10.1029/94JD00483, 1994.

Liang, X., Wood, E. F., and Lettenmaier, D. P.: Surface soil moisture parameterization of the VIC-2L model: Evaluation and modification, Global Planet. Change, 13, 195-206, https://doi.org/10.1016/0921-8181(95)00046-1, 1996.

Liu, Z.: Comparison of Integrated Multi-satellitE Retrievals for GPM (IMERG) and TRMM Multi-satellite Precipitation Analysis (TMPA) Monthly Precipitation Products: Initial Results, J. Hydrometeorol., 17, 777-790, https://doi.org/10.1175/JHM-D15-0068.1, 2016.

Lohmann, D., Nolte-Holube, R., and Raschke, E.: A largescale horizontal routing model to be coupled to land surface parametrization schemes, Tellus A, 48, 708-721, https://doi.org/10.1034/j.1600-0870.1996.t01-3-00009.x, 1996.

McCuen, R. H.: Assessment of Hydrological and Statistical Significance, J. Hydrol. Eng., 21, 2516001, https://doi.org/10.1061/(ASCE)HE.1943-5584.0001340, 2016.

Melsen, L. A., Teuling, A. J., Torfs, P. J. J. F., Uijlenhoet, R., Mizukami, N., and Clark, M. P.: HESS Opinions: The need for process-based evaluation of large-domain hyperresolution models, Hydrol. Earth Syst. Sci., 20, 1069-1079, https://doi.org/10.5194/hess-20-1069-2016, 2016.

Mujumdar, P. P.: Share data on water resources, Nature, 521, 151152,2015

Pai, D. S., Sridhar, L., Rajeevan, M., Sreejith, O. P., Satbhai, N. S., and Mukhopadhyay, B.: Development of a new high spatial resolution $(0.25 \times 0.25)$ long period $(1901-2010)$ daily gridded rainfall data set over India and its comparison with existing data sets over the region, Mausam, 65, 1-18, 2014.

Peng, B., Shi, J., Ni-Meister, W., Zhao, T., and Ji, D.: Evaluation of TRMM Multisatellite Precipitation Analysis (TMPA) Products and Their Potential Hydrological Application at an Arid and
Semiarid Basin in China, IEEE J. Sel. Top. Appl., 7, 3915-3930, https://doi.org/10.1109/JSTARS.2014.2320756, 2014.

Prakash, S., Mitra, A. K., Momin, I. M., Gairola, R. M., Pai, D. S., Rajagopal, E. N., and Basu, S.: A review of recent evaluations of TRMM Multisatellite Precipitation Analysis (TMPA) research products against ground-based observations over Indian land and oceanic regions, MAUSAM, 66, 355-366, 2015.

Prakash, S., Mitra, A. K., AghaKouchak, A., Liu, Z., Norouzi, H., and Pai, D. S.: A preliminary assessment of GPM-based multisatellite precipitation estimates over a monsoon dominated region, J. Hydrol., https://doi.org/10.1016/j.jhydrol.2016.01.029, 2016a.

Prakash, S., Mitra, A. K., Pai, D. S., and AghaKouchak, A.: From TRMM to GPM: How well can heavy rainfall be detected from space?, Adv. Water Resour., 88, 1-7, https://doi.org/10.1016/j.advwatres.2015.11.008, 2016b.

Schumann, A. H.: Thiessen PolygonThiessen polygon BT - Encyclopedia of Hydrology and Lakes, Springer Netherlands, Dordrecht, 648-649, 1998

Shah, H. L. and Mishra, V.: Uncertainty and Bias in Satellitebased Precipitation Estimates over Indian Sub-continental Basins: Implications for Real-time Streamflow Simulation and Flood Prediction, J. Hydrometeorol., 17, 615-636, https://doi.org/10.1175/JHM-D-15-0115.1, 2016.

Srivastava, A. K., Rajeevan, M., and Kshirsagar, S. R.: Development of a high resolution daily gridded temperature data set (1969-2005) for the Indian region, Atmos. Sci. Lett., 10, 249254, https://doi.org/10.1002/asl.232, 2009.

Tang, G., Ma, Y., Long, D., Zhong, L., and Hong, Y.: Evaluation of GPM Day-1 IMERG and TMPA Version-7 legacy products over Mainland China at multiple spatiotemporal scales, J. Hydrol., 533, 152-167, https://doi.org/10.1016/j.jhydrol.2015.12.008, 2016a.

Tang, G., Zeng, Z., Long, D., Guo, X., Yong, B., Zhang, W., and Hong, Y.: Statistical and Hydrological Comparisons between TRMM and GPM Level-3 Products over a Midlatitude Basin: Is Day-1 IMERG a Good Successor for TMPA 3B42V7?, J. Hydrometeorol., 17, 121-137, https://doi.org/10.1175/JHM-D-150059.1, 2016b.

Tawde, S. A. and Singh, C.: Investigation of orographic features influencing spatial distribution of rainfall over the Western Ghats of India using satellite data, Int. J. Climatol., 35, 2280-2293, https://doi.org/10.1002/joc.4146, 2015.

Tong, K., Su, F., Yang, D., and Hao, Z.: Evaluation ofsatellite precipitation retrievals and their potential utilities in hydrologic modeling over the Tibetan Plateau, J. Hydrol., 519, 423-437, https://doi.org/10.1016/j.jhydrol.2014.07.044, 2014.

Wu, H., Adler, R. F., Hong, Y., Tian, Y., and Policelli, F.: Evaluation of Global Flood Detection Using Satellite-Based Rainfall and a Hydrologic Model, J. Hydrometeorol., 13, 1268-1284, https://doi.org/10.1175/JHM-D-11-087.1, 2012.

Wu, X., Xiang, X., Li, L., and Wang, C.: Water level updating model for flow calculation of river networks, Water Sci. Eng., 7, 60-69, https://doi.org/10.3882/j.issn.1674-2370.2014.01.007, 2014.

Ye, A., Duan, Q., Yuan, X., Wood, E. F., and Schaake, J.: Hydrologic post-processing of MOPEX streamflow simulations, J. Hydrol., 508, 147-156, https://doi.org/10.1016/j.jhydrol.2013.10.055, 2014. 
Yong, B., Hong, Y., Ren, L.-L., Gourley, J. J., Huffman, G. J., Chen, X., Wang, W., and Khan, S. I.: Assessment of evolving TRMM-based multisatellite real-time precipitation estimation methods and their impacts on hydrologic prediction in a high latitude basin, J. Geophys. Res., 117, D09108, https://doi.org/10.1029/2011JD017069, 2012.
Zhu, Q., Xuan, W., Liu, L., and Xu, Y.: Evaluation and hydrological application of precipitation estimates derived from PERSIANN CDR, TRMM 3B42V7 and NCEP CFSR over humid regions in China, Hydrol. Process., 30, 3061-3083, https://doi.org/10.1002/hyp.10846, 2016. 